\title{
On Hybrid Ermakov-Painlevé Systems. Integrable Reduction
}

Colin Rogers

To cite this article: Colin Rogers (2017) On Hybrid Ermakov-Painlevé Systems. Integrable Reduction, Journal of Nonlinear Mathematical Physics 24:2, 239-249, DOI: https://doi.org/10.1080/14029251.2017.1313477

To link to this article: https://doi.org/10.1080/14029251.2017.1313477

Published online: 04 January 2021 


\title{
On Hybrid Ermakov-Painlevé Systems. Integrable Reduction
}

\author{
Colin Rogers \\ School of Mathematics and Statistics, The University of New South Wales, Sydney, NSW2052, Australia. \\ c.rogers@unsw.edu.au
}

Received 23 January 2017

Accepted 14 March 2017 Hybrid Ermakov-Painlevé II-IV systems are introduced here in a unified manner. Their admitted Ermakov
invariants together with associated canonical Painlevé equations are used to establish integrability properties.

\section{Introduction}

Prototype hybrid Ermakov-Painlevé II systems were recently introduced via symmetry reduction of an $n+1$-dimensional Manakov-type system in [36]. Two-point Dirichlet boundary value problems for a particular Ermakov-Painlevé II reduction arising out of a Nernst-Planck three-ion electrodiffusion system have been subsequently treated in [2].

Integrable Ermakov-Painlevé II systems with underlying Hamiltonian structure as recently set down in [37] adopt the form

$$
\begin{aligned}
& \ddot{W}_{\mathrm{I}}+\left[\frac{t}{2}+\varepsilon\left(W_{\mathrm{I}}^{2}+W_{\mathrm{II}}^{2}\right)\right] W_{\mathrm{I}}=\frac{1}{W_{\mathrm{I}}^{2} W_{\mathrm{II}}} S^{\prime}\left(W_{\mathrm{II}} / W_{\mathrm{I}}\right), \\
& \ddot{W}_{\mathrm{II}}+\left[\frac{t}{2}+\varepsilon\left(W_{\mathrm{I}}^{2}+W_{\mathrm{II}}^{2}\right)\right] W_{\mathrm{II}}=\frac{1}{W_{\mathrm{I}} W_{\mathrm{II}}^{2}} T\left(W_{\mathrm{I}} / W_{\mathrm{II}}\right)
\end{aligned}
$$

with

$$
\begin{aligned}
& S\left(W_{\mathrm{II}} / W_{\mathrm{I}}\right)=2 \frac{W_{\mathrm{II}}}{W_{\mathrm{I}}} J\left(W_{\mathrm{II}} / W_{\mathrm{I}}\right)+\frac{W_{\mathrm{II}}^{2}}{W_{\mathrm{I}}^{2}} J^{\prime}\left(W_{\mathrm{II}} / W_{\mathrm{I}}\right), \\
& T\left(W_{\mathrm{I}} / W_{\mathrm{II}}\right)=-\frac{W_{\mathrm{II}}^{2}}{W_{\mathrm{I}}^{2}} J^{\prime}\left(W_{\mathrm{II}} / W_{\mathrm{I}}\right),
\end{aligned}
$$

where in the above, a dot denotes a derivative with respect to the independent variable $t$ and the prime denotes a derivative with respect to the argument $W_{\mathrm{II}} / W_{\mathrm{I}}$. The novel Ermakov-NLS systems introduced in [37] admit symmetry reduction to the hybrid Ermakov-Painlevé II system (1.1) via a wave packet ansatz with genesis in a nonlinear optics context [23].

The nonlinear coupled systems as introduced by Ray and Reid in $[34,35]$ have roots in work of Ermakov [20] and adopt the form

$$
\begin{aligned}
& \ddot{W}_{\mathrm{I}}+\omega(t) W_{\mathrm{I}}=\frac{1}{W_{\mathrm{I}}^{2} W_{\mathrm{II}}} S\left(W_{\mathrm{II}} / W_{\mathrm{I}}\right), \\
& \ddot{W}_{\mathrm{II}}+\omega(t) W_{\mathrm{II}}=\frac{1}{W_{\mathrm{II}}^{2} W_{\mathrm{I}}} T\left(W_{\mathrm{I}} / W_{\mathrm{II}}\right) .
\end{aligned}
$$


They admit a distinctive integral of motion and have diverse physical applications, in such areas as nonlinear optics [16, 24, 25, 27,39,40,57], hydrodynamics [41], spinning gas cloud theory [42], magnetogasdynamics [43] and oceanographic warm core eddy theory [44]. Ermakov-Ray-Reid systems also occur in connection with the contribution of orbital angular momentum to the suppression of the collapse of spiralling elliptic solutions in nonlinear Kerr media [17] as well as in the analysis of cloud evolution in a Bose-Einstein condensate [1].

In [45], a 2+1-dimensional version of (1.3) was introduced, while subsequently, multicomponent Ermakov-Ray-Reid systems were derived in a hydrodynamics context via symmetry reduction of a multi-layer fluid model [46]. Therein it was shown that sequences of Ermakov-RayReid systems may be linked by Darboux transformations.

The six classical Painlevé equations $P_{\mathrm{I}}-P_{\mathrm{VI}}$ likewise arise in a wide range of physical applications and play a fundamental role in modern soliton theory (see e.g. Conte [14] and Clarkson [11] together with literature cited therein). Painlevé equations may be shown to possess nonlinear superposition principles associated with the admittance of Bäcklund transformations (see e.g. [26]). Ermakov-Ray-Reid systems likewise admit nonlinear superposition principles albeit of another kind $[34,35]$. Painlevé equations characteristically admit Lax representations while Ermakov-RayReid systems also have been shown to admit underlying linear structure [4].

In general, the studies of Painlevé equations and Ermakov-type systems have proceeded independently. Thus, the only known hybrid solitonic-Ermakov system seems to be that obtained in [54,55] where a 2+1-dimensional Ernst-type system of general relativity as derived in [56], suitably constrained, leads to a novel composition of the integrable 2+1-dimensional sinh-Gordon equation of [30,31] and of a generalised Ermakov-Ray-Reid system. The work of [2,36-38] on ErmakovPainlevé II systems has recently been augmented by the introduction in [47] of prototype ErmakovPainlevé IV systems via a symmetry reduction of a coupled derivative resonant NLS triad. Dirichlet type two-point boundary value problems for a single hybrid Ermakov-Painlevé IV equation have been investigated with regard to existence and uniqueness properties in [3].

The preceding motivates the present work wherein hybrid Ermakov-Painlevé II, ErmakovPainlevé III and Ermakov-Painlevé IV systems are derived in a unified manner. In this context, whereas the classical Ermakov-equation is seen to underlie the standard Ermakov-Ray-Reid system (1.3), the classical Painlevé II - Painlevé IV equations underlie their hybrid Ermakov counterparts. The algorithmic solution of the latter is demonstrated in the case of underlying Hamiltonian-type structure by a combined use of the classical Painlevé II - IV components for the amplitudes of the systems and of admitted Ermakov invariants for the phases.

\section{Extended Ermakov-Ray-Reid Systems}

Here, extended Ermakov-Ray-Reid systems are introduced of the type

$$
\begin{gathered}
\ddot{W}_{\mathrm{I}}-\frac{1}{\Phi}\left[\ddot{\Phi}-\frac{\zeta}{\Phi^{3}}\right] W_{\mathrm{I}}=\frac{1}{W_{\mathrm{I}}^{2} W_{\mathrm{II}}} S\left(W_{\mathrm{II}} / W_{\mathrm{I}}\right), \\
\ddot{W}_{\mathrm{II}}-\frac{1}{\Phi}\left[\ddot{\Phi}-\frac{\zeta}{\Phi^{3}}\right] W_{\mathrm{II}}=\frac{1}{W_{\mathrm{I}} W_{\mathrm{II}}^{2}} T\left(W_{\mathrm{I}} / W_{\mathrm{II}}\right) \\
\zeta \varepsilon \mathbb{R} .
\end{gathered}
$$


The standard Ermakov-Ray-Reid system (1.3) is retrieved in the specialisation when $\Phi$ is determined by the classical Ermakov equation

$$
\ddot{\Phi}+\omega(t) \Phi=\frac{\zeta}{\Phi^{3}} .
$$

It is recalled that the latter admits general solution via a well-known nonlinear superposition principle as readily established by Lie group methods (see e.g. [49,50]). Thus,

$$
\Phi=\sqrt{a x_{1}^{2}+2 c x_{1} x_{2}+b x_{2}^{2}}
$$

where $x_{1}, x_{2}$ are linearly independent solutions of the canonical linear equation

$$
\ddot{W}+\omega(t) W=0
$$

and the constants $a, b$ and $c$ are related by

$$
a b-c^{2}=\frac{\zeta}{\mathscr{W}\left(x_{1} ; x_{2}\right)}
$$

where $\mathscr{W}\left(x_{1} ; x_{2}\right)=x_{1} \dot{x}_{2}-x_{2} \dot{x}_{1}$ is the Wronskian of $x_{1}$ and $x_{2}$. It is noted that the Ermakov-Ray-Reid system (1.3) may be rendered autonomous by the introduction of new dependent and independent variables $\alpha, \beta$ and $z$ according to $[4,46]$

$$
\begin{gathered}
W_{\mathrm{I}}=\alpha(z) U \quad, \quad W_{\mathrm{II}}=\beta(z) U, \\
z=V / U
\end{gathered}
$$

where $U, V$ are linearly independent solutions of (2.4) with unit Wronskian. Thus, under (2.6), reduction of (1.3) is obtained to the autonomous system

$$
\alpha_{z z}=\frac{1}{\alpha^{2} \beta} S(\beta / \alpha) \quad, \quad \beta_{z z}=\frac{1}{\alpha \beta^{2}} T(\alpha / \beta) .
$$

In general, the system (2.1) yields

$$
\begin{aligned}
& \ddot{W}_{\mathrm{I}} \Phi-W_{\mathrm{I}} \ddot{\Phi}+\frac{\zeta W_{\mathrm{I}}}{\Phi^{3}}=\frac{\Phi}{W_{\mathrm{I}}^{2} W_{\mathrm{II}}} S\left(W_{\mathrm{II}} / W_{\mathrm{I}}\right), \\
& \ddot{W}_{\mathrm{II}} \Phi-W_{\mathrm{II}} \ddot{\Phi}+\frac{\zeta W_{\mathrm{II}}}{\Phi^{3}}=\frac{\Phi}{W_{\mathrm{I}} W_{\mathrm{II}}^{2}} T\left(W_{\mathrm{I}} / W_{\mathrm{II}}\right)
\end{aligned}
$$

whence, on setting

$$
\begin{gathered}
\Phi_{\mathrm{I}}=W_{\mathrm{I}} / \Phi \quad, \quad \Phi_{\mathrm{II}}=W_{\mathrm{II}} / \Phi \\
d t^{*}=\Phi^{-2} d t
\end{gathered}
$$

the system (2.1) is reduced to

$$
\begin{gathered}
\Phi_{\mathrm{I}, \mathrm{t}^{*} \mathrm{t}^{*}}+\zeta \Phi_{\mathrm{I}}=\frac{1}{\Phi_{\mathrm{I}}^{2} \Phi_{\mathrm{II}}} S\left(\Phi_{\mathrm{II}} / \Phi_{\mathrm{I}}\right) \\
\Phi_{\mathrm{II}, \mathrm{t}^{*} \mathrm{t}^{*}}+\zeta \Phi_{\mathrm{II}}=\frac{1}{\Phi_{\mathrm{I}} \Phi_{\mathrm{II}}^{2}} T\left(\Phi_{\mathrm{I}} / \Phi_{\mathrm{II}}\right)
\end{gathered}
$$

with independent variable $t^{*}$. 
Here, the integrability of the extended Ermakov-Ray-Reid system (2.1) is addressed with $S\left(W_{\mathrm{II}} / W_{\mathrm{I}}\right)$ and $T\left(W_{\mathrm{I}} / W_{\mathrm{II}}\right)$ parametrised in terms of $J\left(W_{\mathrm{II}} / W_{\mathrm{I}}\right)$ as given by relations (1.2) originally derived in [41] to characterise standard systems (1.3) with underlying Hamiltonian structure. Hence, the extended system (2.1) becomes

$$
\begin{aligned}
& \ddot{W}_{\mathrm{I}}-\frac{1}{\Phi}\left[\ddot{\Phi}-\frac{\zeta}{\Phi^{3}}\right] W_{\mathrm{I}}=\frac{2}{W_{\mathrm{I}}^{3}} J\left(W_{\mathrm{II}} / W_{\mathrm{I}}\right)+\frac{W_{\mathrm{II}}}{W_{\mathrm{I}}^{4}} J^{\prime}\left(W_{\mathrm{II}} / W_{\mathrm{I}}\right), \\
& \ddot{W}_{\mathrm{II}}-\frac{1}{\Phi}\left[\ddot{\Phi}-\frac{\zeta}{\Phi^{3}}\right] W_{\mathrm{II}}=-\frac{1}{W_{\mathrm{I}}^{3}} J^{\prime}\left(W_{\mathrm{II}} / W_{\mathrm{I}}\right)
\end{aligned}
$$

and admits the Ermakov invariant

$$
\mathscr{E}=\frac{1}{2}\left(W_{\mathrm{I}} \dot{W}_{\mathrm{II}}-W_{\mathrm{II}} \dot{W}_{\mathrm{I}}\right)^{2}+\left(\frac{W_{\mathrm{I}}^{2}+W_{\mathrm{II}}^{2}}{W_{\mathrm{I}}^{2}}\right) J\left(W_{\mathrm{II}} / W_{\mathrm{I}}\right) .
$$

Accordingly, the identity

$$
\left(W_{\mathrm{I}}^{2}+W_{\mathrm{II}}^{2}\right)\left(\dot{W}_{\mathrm{I}}^{2}+\dot{W}_{\mathrm{II}}^{2}\right)-\left(W_{\mathrm{I}} \dot{W}_{\mathrm{II}}-W_{\mathrm{II}} \dot{W}_{\mathrm{I}}\right)^{2} \equiv\left(W_{\mathrm{I}} \dot{W}_{\mathrm{I}}+W_{\mathrm{II}} \dot{W}_{\mathrm{II}}\right)^{2}
$$

on use of (2.12) yields

$$
\left(W_{\mathrm{I}}^{2}+W_{\mathrm{II}}^{2}\right)\left(\dot{W}_{\mathrm{I}}^{2}+\dot{W}_{\mathrm{II}}^{2}\right)-2\left[\mathscr{E}-\left(\frac{W_{\mathrm{I}}^{2}+W_{\mathrm{II}}^{2}}{W_{\mathrm{I}}^{2}}\right) J\left(W_{\mathrm{II}} / W_{\mathrm{I}}\right)\right]=\frac{1}{4} \dot{\Sigma}^{2}
$$

where $\Sigma=W_{\mathrm{I}}^{2}+W_{\mathrm{II}}^{2}$. Thus,

$$
\dot{W}_{\mathrm{I}}^{2}+\dot{W}_{\mathrm{II}}^{2}-\frac{2 \mathscr{E}}{\Sigma}+\frac{2 J\left(W_{\mathrm{II}} / W_{\mathrm{I}}\right)}{W_{\mathrm{I}}^{2}}=\frac{1}{4} \dot{\Sigma}^{2} / \Sigma
$$

whence

$$
\dot{W}_{\mathrm{I}} \ddot{W}_{\mathrm{I}}+\dot{W}_{\mathrm{II}} \ddot{W}_{\mathrm{II}}+\mathscr{E}\left(\frac{\dot{\Sigma}}{\Sigma^{2}}\right)+d\left[\frac{J\left(W_{\mathrm{II}} / W_{\mathrm{I}}\right)}{W_{\mathrm{I}}^{2}}\right] / d t=\frac{1}{8}\left[2 \frac{\dot{\Sigma} \ddot{\Sigma}}{\Sigma}-\frac{\dot{\Sigma}^{3}}{\Sigma^{2}}\right] .
$$

But, the system (2.11) shows that

$$
\begin{gathered}
\dot{W}_{\mathrm{I}} \ddot{W}_{\mathrm{I}}+\dot{W}_{\mathrm{II}} \ddot{W}_{\mathrm{II}}-\Delta\left(W_{\mathrm{I}} \dot{W}_{\mathrm{I}}+W_{\mathrm{II}} \dot{W}_{\mathrm{II}}\right)=\left(\frac{2}{W_{\mathrm{I}}^{3}} J+\frac{W_{\mathrm{II}}}{W_{\mathrm{I}}^{4}} J^{\prime}\right) \dot{W}_{\mathrm{I}}-\frac{1}{W_{\mathrm{I}}^{3}} J^{\prime} \dot{W}_{\mathrm{II}} \\
=-d\left[\frac{J\left(W_{\mathrm{II}} / W_{\mathrm{I}}\right)}{W_{\mathrm{I}}^{2}}\right] / d t
\end{gathered}
$$

where

$$
\Delta=\frac{1}{\Phi}\left[\ddot{\Phi}-\frac{\zeta}{\Phi^{3}}\right]
$$

Subtraction of (2.16) and (2.17) now yields

$$
\frac{\mathscr{E}}{\Sigma^{2}}+\frac{\Delta}{2}=\frac{1}{4} \frac{\ddot{\Sigma}}{\Sigma}-\frac{1}{8} \frac{\dot{\Sigma}^{2}}{\Sigma^{2}}=\frac{1}{2} \frac{\left(\Sigma^{\ddot{1} / 2}\right)}{\Sigma^{1 / 2}}
$$

so that, on use of (2.18), a basic Ermakov equation in the ratio $\Sigma^{1 / 2} / \Phi$ results, namely

$$
d^{2}\left(\Sigma^{1 / 2} / \Phi\right) / d t^{* 2}+\zeta\left(\Sigma^{1 / 2} / \Phi\right)=2 \mathscr{E}\left(\Sigma^{1 / 2} / \Phi\right)^{-3}
$$


where the independent variable $t^{*}$ is given by integration of $(2.9)_{3}$. Thus, $\Sigma^{1 / 2} / \Phi$ may be readily obtained by the nonlinear superposition principle admitted by (2.20).

\section{Hybrid Ermakov-Painlevé Systems}

\subsection{The Ermakov-Painlevé II System}

Here, it is required that $\Phi$ be governed by the prototype integrable Ermakov-Painlevé II equation [36]

$$
\ddot{\Phi}+\frac{t}{2} \Phi+\varepsilon \Phi^{3}+\frac{(\alpha-\varepsilon / 2)^{2}}{4 \Phi^{3}}=0 \quad, \quad \varepsilon^{2}=1
$$

whence, on setting $\zeta=-(\alpha-\varepsilon / 2)^{2} / 4$ in (2.1), the system becomes

$$
\begin{aligned}
& \ddot{W}_{\mathrm{I}}+\left[\frac{t}{2}+\varepsilon \Phi^{2}\right] W_{\mathrm{I}}=\frac{1}{W_{\mathrm{I}}^{2} W_{\mathrm{II}}} S\left(W_{\mathrm{II}} / W_{\mathrm{I}}\right), \\
& \ddot{W}_{\mathrm{II}}+\left[\frac{t}{2}+\varepsilon \Phi^{2}\right] W_{\mathrm{II}}=\frac{1}{W_{\mathrm{I}} W_{\mathrm{II}}^{2}} T\left(W_{\mathrm{I}} / W_{\mathrm{II}}\right) .
\end{aligned}
$$

Importantly, as observed in the three-ion electrodiffusion context of [2], in terms of $w=\Phi^{2}$, the Ermakov-Painlevé II equation (3.1) in $\Phi$ delivers the integrable Painlevé XXXIV equation in $w$, namely

$$
\ddot{w}+\frac{\dot{w}^{2}}{2 w}+t w+2 \varepsilon w^{2}+\frac{(\alpha-\varepsilon / 2)^{2}}{2 w}=0 \quad, \quad \varepsilon^{2}=1
$$

while $(2.9)_{3}$ shows that

$$
t^{*}=\int w^{-1} d t
$$

in (2.20).

Here, the system (2.11) becomes

$$
\begin{aligned}
& \ddot{W}_{\mathrm{I}}+\left[\frac{t}{2}+\varepsilon \Phi^{2}\right] W_{\mathrm{I}}=\frac{2}{W_{\mathrm{I}}^{3}} J\left(W_{\mathrm{II}} / W_{\mathrm{I}}\right)+\frac{W_{\mathrm{II}}}{W_{\mathrm{I}}^{4}} J^{\prime}\left(W_{\mathrm{II}} / W_{\mathrm{I}}\right), \\
& \ddot{W}_{\mathrm{II}}+\left[\frac{t}{2}+\varepsilon \Phi^{2}\right] W_{\mathrm{II}}=-\frac{1}{W_{\mathrm{I}}^{3}} J^{\prime}\left(W_{\mathrm{II}} / W_{\mathrm{I}}\right),
\end{aligned}
$$

so that the Ermakov-Painlevé II system (1.1)-(1.2) is retrieved corresponding to the particular solution $\Phi=\Sigma^{1 / 2}$ of the Ermakov equation (2.20) with the relation

$$
\zeta=2 \mathscr{E}=-(\alpha-\varepsilon / 2)^{2} / 4
$$

linking the Painlevé parameter $\alpha$ and Ermakov invariant $\mathscr{E}<0$. It is seen that (2.12) implies that $J\left(W_{\mathrm{II}} / W_{\mathrm{I}}\right)<0$. In this specialisation with $\Phi=\Sigma^{1 / 2},(3.1)$ shows that the amplitude $\Sigma^{1 / 2}=\left(W_{\mathrm{I}}^{2}+\right.$ $\left.W_{\mathrm{II}}^{2}\right)^{1 / 2}$ is governed by an Ermakov-Painlevé II equation directly related with $w=\Sigma$ to the canonical integrable Painlevé XXXIV equation. 
To determine the phase $\Lambda=W_{\mathrm{II}} / W_{\mathrm{I}}$, return is made to the Ermakov invariant relation (2.12) which shows that

$$
\frac{1}{2}\left(\Sigma \frac{d}{d t} \tan ^{-1}\left(\frac{W_{\text {II }}}{W_{\mathrm{I}}}\right)\right)^{2}+\left(1+\left(\frac{W_{\text {II }}}{W_{\mathrm{I}}}\right)^{2}\right) J\left(\frac{W_{\text {II }}}{W_{\mathrm{I}}}\right)=\mathscr{E}
$$

whence, on introduction of the new independent variable $t^{*}$ according to

$$
d t^{*}=\Sigma^{-1} d t
$$

(3.8) shows that

$$
\int \frac{d\left(\tan ^{-1} \Lambda\right)}{\sqrt{2\left(\mathscr{E}-\left(1+\Lambda^{2}\right) J(\Lambda)\right)}}= \pm t^{*}
$$

Once the amplitude $\Sigma^{1 / 2}$ has been determined corresponding to a positive solutions $w=\Sigma$ of Painlevé XXXIV, and the phase $\Lambda$ obtained via (3.9), the original variables $W_{\mathrm{I}}$ and $W_{\mathrm{II}}$ in the Ermakov-Painlevé II system (1.1)-(1.2) are given by the relations

$$
W_{\mathrm{I}}= \pm \sqrt{\frac{\Sigma}{1+\Lambda^{2}}} \quad, \quad W_{\mathrm{II}}= \pm \Lambda \sqrt{\frac{\Sigma}{1+\Lambda^{2}}}
$$

Thus, in summary, the algorithm for generation of solutions of the Ermakov-Painlevé II system (1.1)-(1.2) decomposes into the isolation of solutions $\Sigma>0$ of the integrable Painlevé XXXIV equation (3.3) together with evaluation of the quadrature in (3.9) to determine the phase $\Lambda$.

The importance of positive solutions of Painlevé XXXIV arises, interestingly, elsewhere in the context of boundary value problems for a Painlevé II reduction of the classical two-ion NernstPlanck system $[6,10,15,48]$. Therein, the scaled electric field $\mathrm{E}$ is governed by the canonical integrable Painlevé II equation

$$
E_{z z}=2 E^{3}+z E+\alpha
$$

while the associated ion concentrations are given by

$$
c_{ \pm}= \pm E_{z}+E^{2}=\frac{z}{2}
$$

Thus, on elimination of $\mathrm{E}$ in (3.12), the concentrations $c_{ \pm}$, which by physical considerations are required to be positive, are seen to be governed by the Painlevé XXXIV equation (3.3) with $\varepsilon=-1$. In [6], sequences of exact solutions of this nonlinear equation in terms of Yablonski-Vorob'ev polynomials or classical Airy functions as generated by the iterated action of a Bäcklund transformation were investigated in detail with regard to this positivity constraint. Moreover, in [37], the celebrated Bäcklund transformation for Painlevé II has been recently interpreted at the level of Painlevé XXXIV and used to demonstrate that the problem of integration of the reciprocal of a solution $\Sigma$ of Painlevé XXXIV as required in (3.8) is equivalent to the problem of integration of an associated solution of Painlevé II. The latter problem was then shown to be amenable to solution via the iterated action of the Bäcklund transformation for Painlevé II on a seed solution. 


\subsection{The Ermakov-Painlevé III System}

Painlevé III, namely

$$
w_{z z}=\frac{w_{z}^{2}}{w}-\frac{w_{z}}{z}+\frac{1}{z}\left(\alpha w^{2}+\beta\right)+\gamma w^{3}+\frac{\delta}{w}
$$

where $\alpha, \beta, \gamma$ and $\delta$ are arbitrary parameters has been derived in a wide range of physical contexts, notably in general relativity, electromagnetic radiation and statistical mechanics (see e.g. the literature cited in [33]). It has been derived via symmetry reduction of nonlinear equations in soliton theory, such as the Ernst equations of general relativity [58], the Pohlmeyer-Regge-Lund system [28], the stimulated Raman scattering system [21], as well as Yang-Mills and Bianchi-IX systems in [53] and [18] respectively.

On setting

$$
w=e^{W} \quad, \quad z=e^{t}
$$

in (3.13), it adopts the novel symmetric form

$$
\ddot{W}=\alpha e^{t+W}+\beta e^{t-W}+\gamma e^{2(t+W)}+\delta e^{2(t-W)}
$$

which encapsulates positive solutions of Painlevé III on regions $z>0$. In this connection, it is noted that (3.15) is derived under the similarity transformation

$$
w=e^{W} \quad, \quad z=e^{t}=X T
$$

of the modulated nonlinear hyperbolic equation

$$
W_{X T}=\alpha e^{W}+\beta e^{-W}+X T\left(\gamma e^{2 W}+\delta e^{-2 W}\right)
$$

symmetric in $X$ and $T$. In the case $\beta=\gamma=0$, (3.17) constitutes a modulated Tzitzeica equation and has been derived in the context of affine geometry in [19]. It is remarked that modulated systems arise naturally in continuum mechanics in such areas as the visco-elastodynamics, elastostatics, and elastodynamics of inhomogeneous media (see e.g. [5, 12,29] and literature cited therein). Nonlinear Schrödinger (NLS) equations with modulation also are important, notably in soliton management (Malomed [32]). The structure and application of NLS models with inhomogeneities determined by associated Ermakov-type systems is a subject of current interest and has been recently investigated in $[8,9,36,51,52,59]$.

The above motivates introduction here of hybrid Ermakov-Painlevé III systems with $\Phi$ governed by (3.15), so that,

$$
\ddot{\Phi}=\alpha e^{t+\Phi}+\beta e^{t-\Phi}+\gamma e^{2(t+\Phi)}+\delta e^{2(t-\Phi)}
$$

whence, the system (2.1) becomes

$$
\begin{aligned}
& W_{\mathrm{I}}-\frac{1}{\Phi}\left[\alpha e^{t+\Phi}+\beta e^{t-\Phi}+\gamma e^{2(t+\Phi)}+\delta e^{2(t-\Phi)}-\frac{\zeta}{\Phi^{3}}\right] W_{\mathrm{I}}=\frac{1}{W_{\mathrm{I}}^{2} W_{\mathrm{II}}} S\left(W_{\mathrm{II}} / W_{\mathrm{I}}\right) \\
& W_{\mathrm{II}}-\frac{1}{\Phi}\left[\alpha e^{t+\Phi}+\beta e^{t-\Phi}+\gamma e^{2(t+\Phi)}+\delta e^{2(t-\Phi)}-\frac{\zeta}{\Phi^{3}}\right] W_{\mathrm{II}}=\frac{1}{W_{\mathrm{I}} W_{\mathrm{II}}^{2}} T\left(W_{\mathrm{I}} / W_{\mathrm{II}}\right)
\end{aligned}
$$

If we proceed with the particular solution of the Ermakov equation (2.20) with $\Phi=\Sigma^{1 / 2}$ and $\zeta=2 \mathscr{E}$ where $\mathscr{E}$ is the Ermakov invariant of the system (3.19) then the amplitude $\Sigma^{1 / 2}$ is governed 
by the variant

$$
d^{2} \Sigma^{1 / 2} / d t^{2}=\alpha e^{t+\Sigma}+\beta e^{t-\Sigma}+\gamma e^{2(t+\Sigma)}+\delta e^{2(t-\Sigma)}
$$

of the canonical integrable Painlevé III equation (3.13). In the case of underlying Hamiltonian-type structure, so that $S\left(W_{\mathrm{II}} / W_{\mathrm{I}}\right)$ and $T\left(W_{\mathrm{I}} / W_{\mathrm{II}}\right)$ given by (1.2), the Ermakov-Painlevé III system (3.19) with $\Phi=\Sigma^{1 / 2}$ becomes

$$
\begin{aligned}
\ddot{W}_{\mathrm{I}} & \frac{1}{\Sigma^{1 / 2}}\left[\alpha e^{t+\Sigma^{1 / 2}}+\beta e^{t-\Sigma^{1 / 2}}+\gamma e^{2\left(t+\Sigma^{1 / 2}\right)}+\delta e^{2\left(t-\Sigma^{1 / 2}\right)}-\frac{2 \mathscr{E}}{\Sigma^{3 / 2}}\right] W_{\mathrm{I}} \\
& =\frac{2}{W_{\mathrm{I}}^{3}} J\left(W_{\mathrm{II}} / W_{\mathrm{I}}\right)+\frac{W_{\mathrm{II}}}{W_{\mathrm{I}}^{4}} J^{\prime}\left(W_{\mathrm{II}} / W_{\mathrm{I}}\right), \\
\ddot{W}_{\mathrm{II}} & =\frac{1}{\Sigma^{1 / 2}}\left[\alpha e^{t+\Sigma^{1 / 2}}+\beta e^{t-\Sigma^{1 / 2}}+\gamma e^{2\left(t+\Sigma^{1 / 2}\right)}+\delta e^{2\left(t-\Sigma^{1 / 2}\right)}-\frac{2 \mathscr{E}}{\Sigma^{3 / 2}}\right] W_{\mathrm{II}} \\
& =-\frac{1}{W_{\mathrm{I}}^{3}} J^{\prime}\left(W_{\mathrm{II}} / W_{\mathrm{I}}\right) .
\end{aligned}
$$

The Ermakov integral of motion (2.12) generic to the general hybrid system (2.1) again determines the ratio $\Lambda=W_{\text {II }} / W_{\mathrm{I}}$ via (3.9) but now in the integration of the relation (3.8) to determine $t^{*}$, the squared amplitude $\Sigma$ is determined via (3.20).

It is evident that (3.20) admits the reciprocal invariance

$$
R: \Phi_{R}=-\Phi \quad, \quad \alpha_{R}=-\beta \quad, \quad \beta_{R}=-\alpha \quad, \quad \gamma_{R}=-\delta \quad, \quad \delta_{R}=-\gamma
$$

with induced invariance with $w_{R}=e^{\Phi_{R}}=w^{-1}$ in Painlevé III. This invariance, augmented by a triad of Bäcklund transformations admitted by Painlevé III allows the iterative generation of sequences of its exact solutions and hence of (3.20) via action on appropriate seed solutions. Bäcklund transformations for Painlevé III have been set down in [33] where rational and classical Bessel function solutions are recorded. In the present context, in view of the relations (3.16) interest is restricted to regions $z>0$ on which such solutions are positive. Once solutions $\Sigma^{1 / 2}$ and $\Lambda$ have been determined, in turn, via the integrable Painlevé III variant (3.20) and the integral relation (3.9) derived by means of the Ermakov integral of motion (2.12), the associated solutions $W_{\mathrm{I}}$ and $W_{\mathrm{II}}$ in the hybrid Ermakov-Painlevé III system (3.21) are again given by the relations of the type (3.10).

\subsection{The Ermakov-Painlevé IV System}

In this case, it is required that $\Phi$ be governed by the prototype Ermakov-Painlevé IV equation

$$
\ddot{\Phi}-\left[\frac{3}{4} \Phi^{4}+2 t \Phi^{2}+t^{2}-\alpha\right] \Phi=\frac{\zeta}{\Phi^{3}}
$$

as originally derived in [36] via a symmetry reduction of a coupled derivative NLS system. It is seen that with $w=\Phi^{2}$, the canonical Painlevé IV equation in $w$ is obtained, namely

$$
w \ddot{w}=\frac{1}{2} \dot{w}^{2}+\frac{3}{2} w^{4}+4 t w^{3}+2\left(t^{2}-\alpha\right) w^{2}+\beta .
$$

The latter has applications 'inter alia', in nonlinear lattice theory and the propagation of ion sound waves in plasma physics. It was shown in [13] to arise as a similarity reduction of the classical 
Boussinesq equation, and also occurs, notably, in connection with a symmetry reduction of the discrete Kac-Moerbeke equation [22].

Use of (3.23) in (2.1) produces the hybrid Ermakov-Painlevé IV system

$$
\begin{aligned}
& \ddot{W}_{\mathrm{I}}-\left[\frac{3}{4} \Phi^{4}+2 t \Phi^{2}+t^{2}-\alpha\right] W_{\mathrm{I}}=\frac{1}{W_{\mathrm{I}}^{2} W_{\mathrm{II}}} S\left(W_{\mathrm{II}} / W_{\mathrm{I}}\right), \\
& \ddot{W}_{\mathrm{II}}-\left[\frac{3}{4} \Phi^{4}+2 t \Phi^{2}+t^{2}-\alpha\right] W_{\mathrm{II}}=\frac{1}{W_{\mathrm{I}} W_{\mathrm{II}}^{2}} T\left(W_{\mathrm{I}} / W_{\mathrm{II}}\right) .
\end{aligned}
$$

In the case when $S, T$ are given by the expressions (1.2) and with the particular solution $\Phi=$ $\Sigma^{1 / 2}, \zeta=2 \mathscr{E}$ of the Ermakov equation (2.20), the solutions $W_{\mathrm{I}}, W_{\text {II }}$ are given by the expressions (3.10) where now $\Sigma$ therein is determined by the Ermakov-Painlevé IV equation

$$
d^{2} \Sigma^{1 / 2} / d t^{2}-\left[\frac{3}{4} \Sigma^{2}+2 t \Sigma+t^{2}-\alpha\right] \Sigma^{1 / 2}=\frac{2 \mathscr{E}}{\Sigma^{3 / 2}} .
$$

In the latter connection, it is remarked that a privileged sequence of bound state solutions which are non-negative may be generated by the iterated action of a Bäcklund transformation and corresponds to parameters $\alpha$ an odd integer and $\beta=0$ (see [7]). The ratio $\Lambda=W_{\mathrm{I}} / W_{\mathrm{II}}$ is again determined via the relations (3.10) but where now $\Sigma$ is a positive solution of Painlevé IV.

\section{References}

[1] J. Abdullaev, A.S. Desyatnikov and E.A. Ostravoskaya, Suppression of collapse for matter waves with orbital angular momentum, J. Opt. 13 (2011) 064023.

[2] P. Amster and C. Rogers, On a Ermakov-Painlevé II reduction in three-ion electrodiffusion. A Dirichlet boundary value problem, Discrete and Continuous Dynamical Systems 35 (2015) 3277-3292.

[3] P. Amster and C. Rogers, On Dirichlet two-point boundary value problems for the Ermakov-Painlevé IV equation, J. Applied Math. \& Computing 48 (2015) 71-81.

[4] C. Athorne, C. Rogers, U. Ramgulam and A. Osbaldestin, On linearisation of the Ermakov system, Phys. Lett. A 143 (1990) 207-212.

[5] D.W. Barclay, T.B. Moodie and C. Rogers, Cylindrical impact waves in inhomogeneous Maxwellian visco-elastic media, Acta Mechanica 29 (1978) 93-117.

[6] L.K. Bass, J. Nimmo, C. Rogers and W.K. Schief, Electrical structures of interfaces. A Painlevé II model, Proc. Roy. Soc. Lond. A466 (2010) 2117-2136.

[7] A.P. Bassom, P.A. Clarkson, A. C. Hicks and J.B. McLeod, Integral equations and exact solutions for the fourth Painlevé equation, Proc. Roy. Soc. Lond. A437 (1992) 1-24.

[8] J. Belmonte-Beita, V.M. Pérez-Garcia and V. Vekslechik, Lie symmetries and solitons in nonlinear systems with spatially inhomogeneous nonlinearities, Physical Review Letters 98 (2007) 064102.

[9] J. Belmonte-Beita, V.M. Pérez-Garcia, V. Vekslechik and V.V. Konotop, Localised nonlinear waves in systems with time and space-modulated nonlinearities, Physical Review Letters 100 (2008) 164102.

[10] A.J. Bracken, L. Bass and C. Rogers, Bäcklund flux quantization in a model of electrodiffusion based on Painlevé II, J. Phys. A: Math. \& Theor. 45 (2012) 105204.

[11] P.A. Clarkson, Painlevé equations. Nonlinear special functions, J. Comput. Appl. Math. 153 (2003) 127-140.

[12] D.L. Clements, C. Atkinson and C. Rogers, Antiplane crack problems for an inhomogeneous elastic material, Acta Mechanica 29 (1978) 199-211.

[13] P.A. Clarkson and M.D. Kruskal, New similarity solutions of the Boussinesq equation, J. Math. Phys. 30 (1989) 2201-2213.

[14] R. Conte (Ed.), The Painlevé Property: One Century Later (Springer, New York, 1999).

[15] R. Conte, C. Rogers and W.K. Schief, Painlevé structure of a multi-ion electrodiffusion system, J. Phys. A: Math. \& Theor. 40 (2007) F1031-F1040. 
[16] F. Cornolti, M. Lucchesi and B. Zambon, Elliptic Gaussian beam self-focussing in nonlinear media, Opt. Commun. 75 (1990) 129-135.

[17] A.S. Desyatnikov, D. Buccoliero, M.R. Dennis and Y.S. Kivshar, Suppression of collapse for spiralling elliptic solitons, Phys. Rev. Lett. 104 (2010) 053902-1-053902-4.

[18] K.P. Dod, A comment on a paper by Pederson and Poon, Class. Quantum Gravity 8 (1991) 1049-1051.

[19] M. Dunajski and P. Prim, Plansangkate, Strominger-Yau-Zaslow geometry. Affine spheres and Painlevé III, Commun. Math. Phys. 290 (2009) 997-1024.

[20] V.P. Ermakov, Second-order differential equations: conditions of complete integrability, Univ. Izy. Kiev 20 (1880) 1-25.

[21] A.S. Fokas and C.R. Menyuk, Integrability and self-similarity in transient stimulated Raman scattering, J. Nonlinear Science 9 (1999) 1-31.

[22] A.S. Fokas, A.R. Its and A.V. Kitaev, Discrete Painlevé equations and their appearance in quantum gravity, Commun. Math. Phys. 142 (1991) 313-344.

[23] J.A. Giannini and R.I. Joseph, The role of the second Painlevé transcendent in nonlinear optics, Phys. Lett. A 141 (1989) 417-419.

[24] A.M. Goncharenko, Y.A. Logvin, A.M. Samson, P.S. Shapovalev and S.I. Turovets Ermakov Hamiltonian systems in nonlinear optics of elliptic Gaussian beams, Phys. Lett. A 160 (1991) 138-142.

[25] A.M. Goncharenko, Y.A. Logvin, A.M. Samson and P.S. Shapovalov, Rotating ellipsoidal gaussian beams in nonlinear media, Opt. Commun. 81 (1991) 225-230.

[26] V.I. Gromak and G. Filipuk, On the transformations of the sixth Painlevé equation J. Nonlinear Math. Phys. 10 (2003) 57-68.

[27] C.R. Guiliano, J.H. Marburger and A. Yariv, Enhancement of self-focussing threshold in sapphire with elliptical beams, Appl. Phys. Lett. 21 (1972) 58-60.

[28] M. Jimbo, An inverse scattering problem and the fifth Painlevé transcendent, Prog. Theor. Phys. 61 (1979) 359-360.

[29] F.C. Karal and J.B. Keller, Elastic wave propagation in homogeneous and inhomogeneous media, $J$. Acoust. Soc. America 31 (1959) 694-705.

[30] B. Konopelchenko and C. Rogers, On 2+1-dimensional nonlinear systems of Loewner-type, Phys. Lett. A 158 (1991) 391-397.

[31] B. Konopelchenko and C. Rogers, On generalised Loewner systems: novel integrable equations in 2+1dimensions, J. Math. Phys. 34 (1993) 214-242.

[32] B.A. Malomed, Soliton Management in Periodic Systems (Springer, New York, 2006).

[33] A.E. Milne and P.A. Clarkson, Rational solutions and Bäcklund transformations for the third Painlevé equation, in Applications of Analytic and Geometric Methods to Nonlinear Differential Equations, Ed. P.A. Clarkson, Nato ASI Series, Mathematical and Physical Sciences, Kluwer, Vol. 413 (1993) 341352.

[34] J.R. Ray, Nonlinear superposition law for generalised Ermakov systems, Phys. Lett. A 78 (1980) 4-6.

[35] J.L. Reid and J.R. Ray, Ermakov systems, nonlinear superposition and solution of nonlinear equations of motion, J. Math. Phys. 21 (1980) 1583-1587.

[36] C. Rogers, A novel Ermakov-Painlevé II system: n+1-dimensional coupled NLS and elastodynamic reductions, Stud. Appl. Math. 133 (2014) 214-231.

[37] C. Rogers and W.K. Schief, On Ermakov-Painlevé II systems. Integrable reduction, Meccanica 51 (2016) 2967-2974.

[38] C. Rogers and W.K. Schief, On a novel resonant Ermakov-NLS system: Painlevé reduction, submitted (2016).

[39] C. Rogers, B. Malomed, K. Chow and H. An, Ermakov-Ray-Reid systems in nonlinear optics, J. Phys. A: Math. Theor. 43 (2010) 455214.

[40] C. Rogers, B. Malomed and H. An, Ermakov-Ray-Reid reductions of variational approximations in nonlinear optics, Stud. Appl. Math. 129 (2012) 389-413.

[41] C. Rogers and H. An, Ermakov-Ray-Reid systems in 2+1-dimensional rotating shallow water theory, Stud. Appl. Math. 125 (2010) 275-299. 
[42] C. Rogers and W.K. Schief, On the integrability of a Hamiltonian reduction of a 2+1-dimensional nonisothermal rotating gas cloud system, Nonlinearity 24 (2011) 3165-3178.

[43] C. Rogers and W.K. Schief, The pulsrodon in 2+1-dimensional magneto-gasdynamics. Hamiltonian structure and integrability, J. Math. Phys. 52 (2011) 083701.

[44] C. Rogers, Elliptic warm-core theory: the pulsrodon, Phys. Lett. 138A (1989) 267-273.

[45] C. Rogers, C. Hoenselaers and J.R. Ray, On 2+1-dimensional Ermakov systems, J. Phys. A: Math. Gen. 26 (1993) 2625-2633.

[46] C. Rogers and W.K. Schief, Multi-component Ermakov systems: structure and linearization, J. Math. Anal. Appl. 198 (1996) 194-220.

[47] C. Rogers, Hybrid Ermakov-Painlevé IV systems, J. Nonlinear Mathematical Physics 21 (2014) 628642.

[48] C. Rogers, A.P. Bassom and W.K. Schief, On a Painlevé II model in steady electrolysis: application of a Bäcklund transformation, J. Math. Anal. Appl. 240 (1999) 367-381.

[49] C. Rogers and U. Ramgulam, A nonlinear superposition principle and Lie group invariance: application in rotating shallow water theory, Int. J. Nonlinear Mech. 24 (1989) 229-236.

[50] C. Rogers, W.K. Schief and P. Winternitz, Lie-theoretical generalization and discretization of the Pinney equation, J. Math. Anal. Appl. 216 (1997) 246-264.

[51] C. Rogers, Gausson-type representations in nonlinear physics. Ermakov modulation, Physica Scripta 89 (2014) 105208.

[52] C. Rogers, G. Saccomandi and L. Vergori, Ermakov-modulated nonlinear Schródinger models: integrable reduction, J. Nonlinear Mathematical Physics 23 (2016) 108-126.

[53] C.K. Saclioglu, Liouville and Painlevé equations and Yang-Mills strings, J. Math. Phys. 25 (1984) 3214-3220.

[54] W.K. Schief and C. Rogers, On a Laplace sequence of nonlinear integrable Ernst-type equations, Algebraic Aspects of Integrable Systems, Progress in Nonlinear Differential Equations (Eds. A. Fokas \& I.M. Gelfand), Birkhauser 26 (1996) 315-321.

[55] W.K. Schief, C. Rogers and A. Bassom, Ermakov systems of arbitrary order and dimension: structure and linearization, J. Phys. A: Math. Gen. 29 (1996) 903-911.

[56] W.K. Schief, On a 2+1-dimensional integrable Ernst-type equation, Proc. Roy. Soc. London A466 (1994) 381-398.

[57] W.G. Wagner, H.A. Haus and J.H. Marburger, Large-scale self-trapping of optical beams in the paraxial ray approximation, Phys. Rev. 175 (1968) 256-266.

[58] P. Wils, A new Painlevé solution of the Ernst equations, Phys. Lett. A 135 (1989) 425-427.

[59] J.F. Zhang, Yi S. Li, J. Meng, L. Wo and B.A. Malomed, Matter-wave solitons and finite-amplitude Bloch waves in optical lattices with a spatially modulated nonlinearity, Phys. Rev. A 82 (2010) 033614. 